\title{
Regenerative Virtual Therapy: The Use of Multisensory Technologies and Mindful Attention for Updating the Altered Representations of the Bodily Self
}

\author{
Giuseppe Riva ${ }^{1,2,3 * t}$, Silvia Serino ${ }^{2,3+}$, Daniele Di Lernia ${ }^{2,3+}$ and Francesco Pagnini $i^{3,4+}$ \\ ${ }^{1}$ Applied Technology for Neuro-Psychology Laboratory, Istituto Auxologico Italiano, Milan, Italy, ${ }^{2}$ Humane Technology \\ Laboratory, Università Cattolica del Sacro Cuore, Milan, Italy, ${ }^{3}$ Department of Psychology, Università Cattolica del Sacro \\ Cuore, Milan, Italy, ${ }^{4}$ Department of Psychology, Harvard University, Cambridge, MA, United States
}

OPEN ACCESS

Edited by:

Melanie Krüger,

Leibniz University of Hannover,

Germany

Reviewed by:

Lucie Bréchet,

University of Geneva, Switzerland

Lilian Aline Weber,

ETH Zürich, Switzerland

*Correspondence:

Giuseppe Riva

giuseppe.riva@unicatt.it

tORCID:

Giuseppe Riva

orcid.org/0000-0003-3657-106X

Silvia Serino

orcid.org/0000-0002-8422-1358

Daniele Di Lernia

orcid.org/0000-0001-6850-6866

Francesco Pagnin

orcid.org/0000-0003-1612-4211

Received: 29 July 2021 Accepted: 04 October 2021 Published: 03 November 2021

Citation:

Riva G, Serino S, Di Lernia D and Pagnini F (2021) Regenerative Virtual

Therapy: The Use of Multisensory Technologies and Mindful Attention for Updating the Altered Representations of the Bodily Self. Front. Syst. Neurosci. 15:749268. doi: 10.3389/fnsys.2021.749268
The term "regenerative medicine" (RM) indicates an emerging trend in biomedical sciences that aims at replacing, engineering, or regenerating human cells, tissues, or organs to restore or establish normal function. So far, the focus of RM has been the physical body. Neuroscience, however, is now suggesting that mental disorders can be broadly characterized by a dysfunction in the way the brain computes and integrates the representations of the inner and outer body across time [bodily self-consciousness (BSC)]. In this perspective, we proposed a new kind of clinical intervention, i.e., "Regenerative Virtual Therapy" (RVT), which integrates knowledge from different disciplines, from neuroscience to computational psychiatry, to regenerate a distorted or faulty BSC. The main goal of RVT was to use technology-based somatic modification techniques to restructure the maladaptive bodily representations behind a pathological condition. Specifically, starting from a Bayesian model of our BSC (i.e., body matrix), we suggested the use of mindful attention, cognitive reappraisal, and brain stimulation techniques merged with high-rewarding and novel synthetic multisensory bodily experience (i.e., a virtual reality full-body illusion in sync with a low predictabllity interoceptive modulation) to rewrite a faulty experience of the body and to regenerate the wellbeing of an individual. The use of RVT will also offer an unprecedented experimental overview of the dynamics of our bodily representations, allowing the reverse-engineering of their functioning for hacking them using advanced technologies.

Keywords: embodiment (and its derivatives), multisensory integration, Bayesian surprise maximization, bodily full-body illusions, brain stimulation, interoceptive technology, virtual reality, mindfulness

\section{INTRODUCTION}

Holmes et al. (2014) published, in Nature, the article "Psychological treatments: A call for mentalhealth science," calling for an alliance between clinicians and neuroscientists to advance our understanding of psychological treatments. They underlined that "we do not yet fully understand how psychological therapies work-or when they don't. Neuroscience is shedding light on how

Abbreviations: AR, autobiographical recall; BSC, bodily self-consciousness; GVS, galvanic vestibular stimulation; PAF, positive affective forecasting; RCT, randomized controlled trial; RVT, regenerative virtual therapy; tDCS, transcranial direct current stimulation; TMS, transcranial magnetic stimulation; VNS, vagus nerve stimulation. 
to modulate emotion and memory, habit, and fear learning. But psychological understanding and treatments have, as yet, profited much too little from such developments." (p. 288) A key problem underlying most, if not all, psychopathologies is schema rigidity (Morris and Mansell, 2018): many individuals are unable to avoid and update automatic beliefs and behaviors that rely on preexisting or underlying assumptions and evaluations that might not apply to the current situation with significant negative effects.

However, recent key discoveries in neuroscience are outlining a new conceptual framework, merging the embodied cognition approach (Clark, 2016b; Newen, 2018) with the predictive brain hypothesis (Friston, 2010; Owens et al., 2018), on how selfschemas influence the psychological functioning that directly links them to the processing of multisensory bodily signals (Blanke et al., 2015; Riva, 2018). Paulus et al. (2019) recently explained that "these conceptual models suggest that mental disorders can be broadly characterized by a dysfunction in the way the brain computes and integrates representations of the inner and outer worlds of the body across time. According to this view, changes in mood and anxiety are a by-product of the brain's biased translation of what it expects will happen versus what is actually happening in these worlds, producing a persistent discrepancy/error signal when outcomes are observed." (p. 99).

Following this vision, in the last decade, several mental health conditions have been associated with damage and/or malfunctioning of the bodily self, i.e., eating and weight disorders (Riva and Gaudio, 2012; Keizer et al., 2013; Dakanalis et al., 2016; Scarpina et al., 2016; Riva and Dakanalis, 2018), depression (Barrett et al., 2016), schizophrenia (Postmes et al., 2014; Klaver and Dijkerman, 2016; Ferri et al., 2017; Möller et al., 2021), autism (Ropar et al., 2018; Riva et al., 2019a), and chronic pain (Tsay et al., 2015; Di Lernia et al., 2016).

Nevertheless, since the study by Holmes et al. (2014), things have not changed significantly: these basic research discoveries have not yet met a direct clinical application. While the change mechanisms of successful psychotherapeutic approaches, such as the current gold standard for many mental diseases, i.e., cognitive behavioral therapy (CBT), are often based on schema modifications, they do not target directly with their methods all the components of a faulty bodily experience.

In 2014, the first author of this perspective suggested in a letter to Nature (Gaggioli and Riva, 2014) that the use of technology, and in particular virtual reality (Riva et al., 2019b), could be a possible solution to this problem, offering a powerful tool for improving evidence-based psychological treatments. More recently, the two different studies by Browning et al. (2020) and Nair et al. (2020) suggested the use of computational characterizations/assays of behavior for patients undergoing psychological therapies using mathematical/Bayesian models of key cognitive processes.

In this perspective, we wanted to follow both suggestions by introducing a new therapeutical approach, i.e., Regenerative Virtual Therapy (RVT). Specifically, starting from a Bayesian model of our bodily self (i.e., body matrix), we suggested the use of mindful attention, cognitive reappraisal, and brain stimulation techniques merged with high-rewarding and novel synthetic multisensory bodily experience to rewrite a faulty bodily experience and to regenerate the wellbeing of an individual.

\section{FROM REGENERATIVE MEDICINE TO REGENERATIVE VIRTUAL THERAPY}

In medicine, a profound paradigm shift was introduced by regenerative medicine ( $\mathrm{RM})$, an emerging trend in biomedical sciences that aims at "replacing, engineering, or regenerating human cells, tissues, or organs to restore or establish normal function" (Mason and Dunnill, 2008). The fundamental value of RM is the possibility to regenerate the organism and to force the body to heal itself. RM allows not only to better cope with the symptoms but also to eradicate the cause of the symptoms by helping the body to restore the damaged cells to a healthy state (Mason and Dunnill, 2008; Mahla, 2016).

So far, the focus of RM has been the physical body: human stem cells and biomolecular therapies are used to restore the normal structure and function of a missing or damaged organ. However, the abovementioned evidence from recent neuroscientific discoveries suggests that by exploiting the mechanisms of the "predictive brain," it is also possible to regenerate our bodily experience [i.e., bodily self-consciousness (BSC)].

The BSC represents a challenging research field because it requires an interdisciplinary framework to provide a link between all the afferent levels and brain circuits involved in a particular bodily experience (Lux et al., 2021). However, recent neuroscience research (Blanke, 2012; Blanke et al., 2015; Riva, 2018; Park and Blanke, 2019) is shedding new light on the processes involved in the BSC.

Even if BSC is experienced by the individual as a unitary perception, neuroimaging and neurological data suggested that BSC includes different layers (Figure 1) that integrate both sensory and cognitive bodily data in a coherent experience (Moseley et al., 2012; Apps and Tsakiris, 2014; Riva, 2018).

Specifically, the layers are organized around a bidirectional hierarchical structure following the active inference and predictive coding paradigms introduced by the Bayesian brain theory (Apps and Tsakiris, 2014; Friston et al., 2014). The result of this process is a coarse supramodal multisensory representation of the body and the space around it (i.e., body matrix, refer to Figure 1), emerging from the flow of information across large-scale networks that link various regions of the brain (Moseley et al., 2012; Gallace and Spence, 2014; Sedda et al., 2016; Riva, 2018).

First, the Bayes' theorem (Manjaly and Iglesias, 2020) explains how an initial representation/layer (or "prior," a prediction based on a model of the environment and the body) is integrated with or updated by new observations (i.e., sensory input), resulting in an updated representation (or posterior probability). In this view, the possibility of updating a prior depends on the so-called prediction error, or "surprise" (Stephan et al., 2016): the discrepancy between new data and prior belief (i.e., predicted sensory information), which is weighted by the ratio 


\section{Sensory signals from outside the body (Perception)}

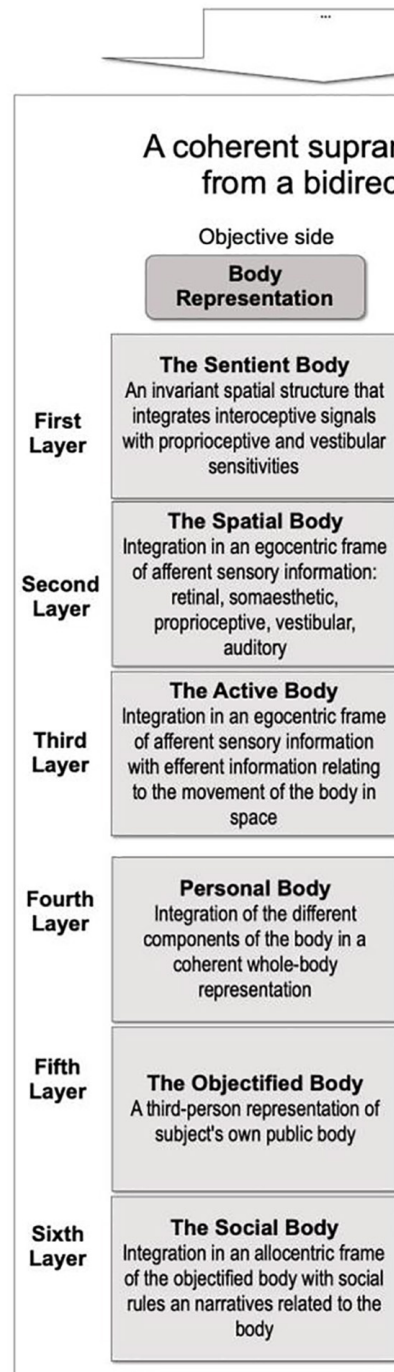

\section{Body Matrix}

coherent supramodal representation of the body resulting from a bidirectional hierarchical predictive structure
The Sentient Body An invariant spatial structure that rates interoceptive signals sensitivities

The Spatial Body tegration in an egocentric frame ent sensory information:

Active Body ent sensory information rent information relating ment of the body in Integration of the different coherent whole-body representation
Personal Body

\section{Sensory signals from inside the body (Interoception, Proprioception, Vestibular Input)}

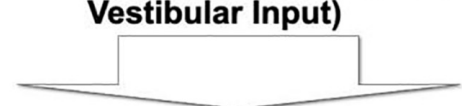

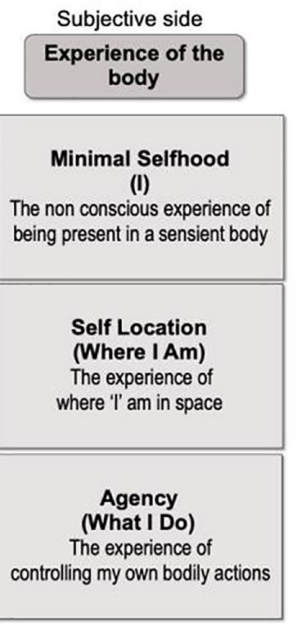

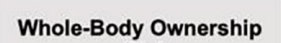
(Me)

The first-person reflective experience of owning a whole body

Objectified self (Mine)

The objectified sense of what belongs to the self including the reflective experience of being exposed and visible to others

\section{Body Satisfaction} (Ideal Me)

The experience of having a body in agreement/disagreement with social norms

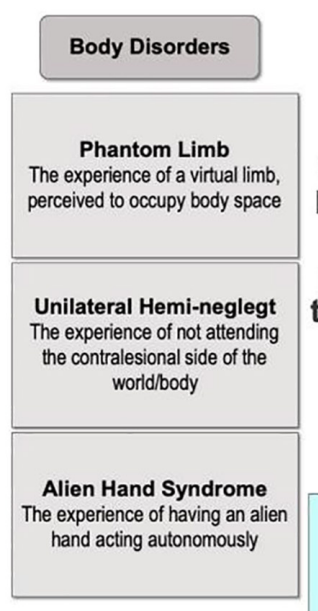

Autoscopic Phenomena The experience of seeing a second own body in the extrapersonal space

Xenomelia

The non-acceptance of one's own extremities and the resulting desire for elective limb amputation

Body Dysmorphia The experience of falsely believing of having something wrong with a specific part of the body
These representations are organized around a bidirectional hierarchical predictive structure

\section{Prediction errors from lower layers are propagated to the higher ones<smiles>C1CCCC1</smiles>

\section{The structure is adjusted so that top-down predictions cancel prediction errors at the lower layers

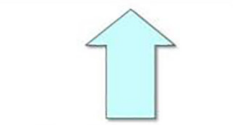

Higher levels provide priors to the layers below.

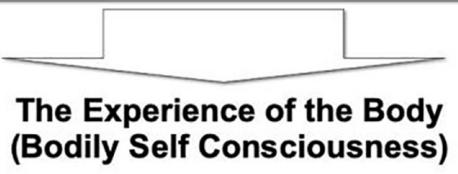

FIGURE 1 | The body matrix.

between data precision (the confidence one assigns to the data) and prior representation precision (the confidence one assigns to a prior belief). In this view, when the precision of the data (i.e., likelihood) is higher than the precision of the prior representation, a large update results i.e., (the posterior moves more strongly toward the data Manjaly and Iglesias, 2020). Simply speaking, precise priors reduce, and precise sensory data increase the probability of a representation update.
Moreover, in a bidirectional hierarchical structure similar to the one used by BSC, higher levels provide priors to the level below, and these constraints are progressively tuned by the sensory input coming from the lower levels (Apps and Tsakiris, 2014; Clark, 2016a). Specifically, prediction errors are propagated to the higher level to adjust the structure of the model so that the top-down predictions cancel prediction errors at the lower level (Clark, 2016a). In this 
view, the greater is the prediction error at the bottom of the hierarchy (i.e., ascending prediction error), the further up the hierarchy its effects will percolate and lead to the adjustments of the model (Manjaly and Iglesias, 2020). In brief, the minimization of prediction errors involves a reciprocal exchange of signals between hierarchical levels: prediction errors ascend the hierarchy to revise expectations, which generate descending predictions that resolve or suppress prediction errors at the level below.

These principles and different recent studies suggest that it is possible to update the contents of our experience of the body both at a low level, i.e., proprioception and interoception (Henriques and Cressman, 2012; Nourouzpour et al., 2015; Di Lernia et al., 2018), and a high level, i.e., social cognition and self-identification (Tajadura-Jiménez and Tsakiris, 2014; Maister et al., 2015), using advanced technological tools. In the below sections, we further detailed the contents of our proposal: the use of technologybased somatic modification techniques to facilitate a potential revision of maladaptive predictions (priors). Specifically, we planned to use mindful attention, cognitive reappraisal, and brain stimulation techniques merged with high-rewarding and novel synthetic multisensory bodily experiences such as virtual reality bodily illusions.

\section{REGENERATIVE VIRTUAL THERAPY TECHNOLOGY}

Since the discovery of the rubber hand illusion (Botvinick and Cohen, 1998) and the emergence of non-invasive brain stimulation methodologies (Tatti et al., 2016), different researchers have used advanced technologies to alter body perceptions in clinical and non-clinical populations. In particular, three different approaches have been developed as follows:

1. Virtual bodily illusions (Matamala-Gomez et al., 2021), also known as full-body ownership illusions, use virtual reality technologies to trick the predictive coding mechanisms of the brain, thereby inducing users a sense of ownership over a virtual body.

2. Interoceptive technologies (Schoeller et al., 2019), modulate interoceptive signals. They include technologies for producing a direct modulation of interoceptive signals [i.e., c-fibers stimulation, Björnsdotter et al. (2010); Di Lernia et al. (2020); or sonoception, Wiederhold and Riva (2019)] and technologies generating illusions by providing false feedback of the physiological states of individuals (Iodice et al., 2019).

3. Brain stimulation techniques, for example, transcranial direct current stimulation (tDCS) and transcranial magnetic stimulation (TMS) (Avenanti et al., 2018; Mancuso et al., 2020; Stramba-Badiale et al., 2020), and also vagus nerve stimulation (Neuser et al., 2020) and galvanic vestibular stimulation (Ponzo et al., 2018, 2019) modify both bottom-up (Pollatos et al., 2016) and top-down (Marotta et al., 2021) bodily signals.
Existing studies, however, suggest that the effects of the abovementioned approaches on higher cognitive processes are temporary, even with non-pathological individuals. For example, as reported by Freeman et al. (2017), the longest followup in studies with virtual bodily illusions for correcting the perception of the body in participants with eating disorders is just $2 \mathrm{~h}$ (Keizer et al., 2016). In our opinion, this can be explained by the bidirectional hierarchical predictive structure used by BSC. In this structure, the minimization of prediction errors involves a reciprocal exchange of signals between hierarchical layers: prediction errors ascend the hierarchy to revise expectations, which generate descending predictions that resolve or suppress prediction errors at the level below. In this view, generating prediction errors in one layer is not enough to guarantee a revision on higher levels, producing the long-term modification of the BSC.

Following a prediction error, the contents of the body matrix are adjusted in evaluating the (dis)agreement between the perceived sensory activity, and the body experience predicted through the integration of contents from different bodily and cognitive representations (Talsma, 2015). Among others, three possible effects can be activated (Pezzulo et al., 2015; Owens et al., 2018; Mirza et al., 2019), namely, (1) prediction errors ascend the cortical hierarchy to change predictions (model updating), (2) predictions selectively sample sensory input to change the sensations being predicted to agree with their content (active inference) through action and/or attentional shifts, or (3) attention is used to optimize the precision afforded to different parts of the sensorium.

In general, prediction errors in a bidirectional hierarchical predictive structure generate a model updating only when:

- The extent of the prediction errors is high: As we have noticed before, the greater is the prediction error, the further up the hierarchy its effects will percolate and lead to model adjustments (Manjaly and Iglesias, 2020). In general, prediction errors arise from the lower layers because they are easier to control and modify. However, errors can be generated in any of the layers of the body matrix (Clark, 2013).

- The precision of the prediction errors is high: During multisensory integration, bottom-up bodily signals from different sensory modalities and top-down predictions are weighted according to their contextual reliability and combined to produce a unitary experience of the body. This precision-weighting mechanism is critical for balancing appropriately prediction and sensory stimuli (Barca and Pezzulo, 2020): if it is wrong and it assigns to bottom-up sensory stimuli a low precision, the generated prediction error does not produce an update. Precision operates both within and between modalities, and it is improved both by the level of attention provided to the specific signal (Smout et al., 2019) and by reducing the noise of the sensory signal (Pezzulo et al., 2015).

- The surprise of the prediction errors is high: The results of a study by McGuire et al. (2014) suggest that the 
level of surprise is related to three computationally and neuroanatomically distinct factors.

- The first one is the extent of the prediction error. The more the outcome is particularly unpredictable or surprising under the current model, the more is the probability of an update.

- The second one is the relative uncertainty of the current model. The higher is the level of uncertainty of the model, the higher is the probability of an update.

- The third one is the level of reward. The higher is the potential reward produced by the update, the higher is its probability. Possible rewards are a better image of the self or a positive emotional state.

In this view, the main goal of RVT was to allow a potential revision and de-weighting of maladaptive predictions through the integration of different technology-based somatic modification techniques with mindfulness and cognitive reappraisal. The suggested process is as follows (Figure 2):

1. The development of a synthetic multisensory experience (visuotactile and interoceptive) able to generate significant prediction errors, for contrasting the dysfunctional internal model: to reach this goal, we planned to use a virtual reality full-body illusion in a body different from the real one (i.e., an anorectic subject in a normal body) in sync with an interoceptive modulation. During the experience, when the experimenter strokes the participant on the abdomen, delivering a tactile stimulation, he/she observes this stroking on the abdomen of the avatar (visuotactile sync). The same happens during interoceptive stimulation: any stroke on the real hand is synched to the virtual one.

2. The use of brain stimulation techniques to reduce the influence of top-down predictions: to manipulate the process of precision-weighting, we suggested the use of tDCS to disrupt the prediction processes and to reduce the influence of top-down predictions. Avenanti et al. used both cathodal (inhibitory) and anodal (excitatory) tDCS over the left inferior frontal cortex, a key area of the action observation network involved in coupling actionperception with execution, during an action prediction task (Avenanti et al., 2018). Their results preliminarily suggest that down- and upregulating excitability using tDCS can hinder and enhance action prediction abilities, respectively.

3. The use of mindfulness attention to improving the precision of the synthetic multisensory experience: mindful attention (Papies et al., 2015) is defined as a form of attention that can increase the salience of the moment-by-moment experience and reduce the impact of predefined schemas. When mindfully attentive, people become aware of thoughts and experiences, observing them as transient mental events (Bennett et al., 2021).
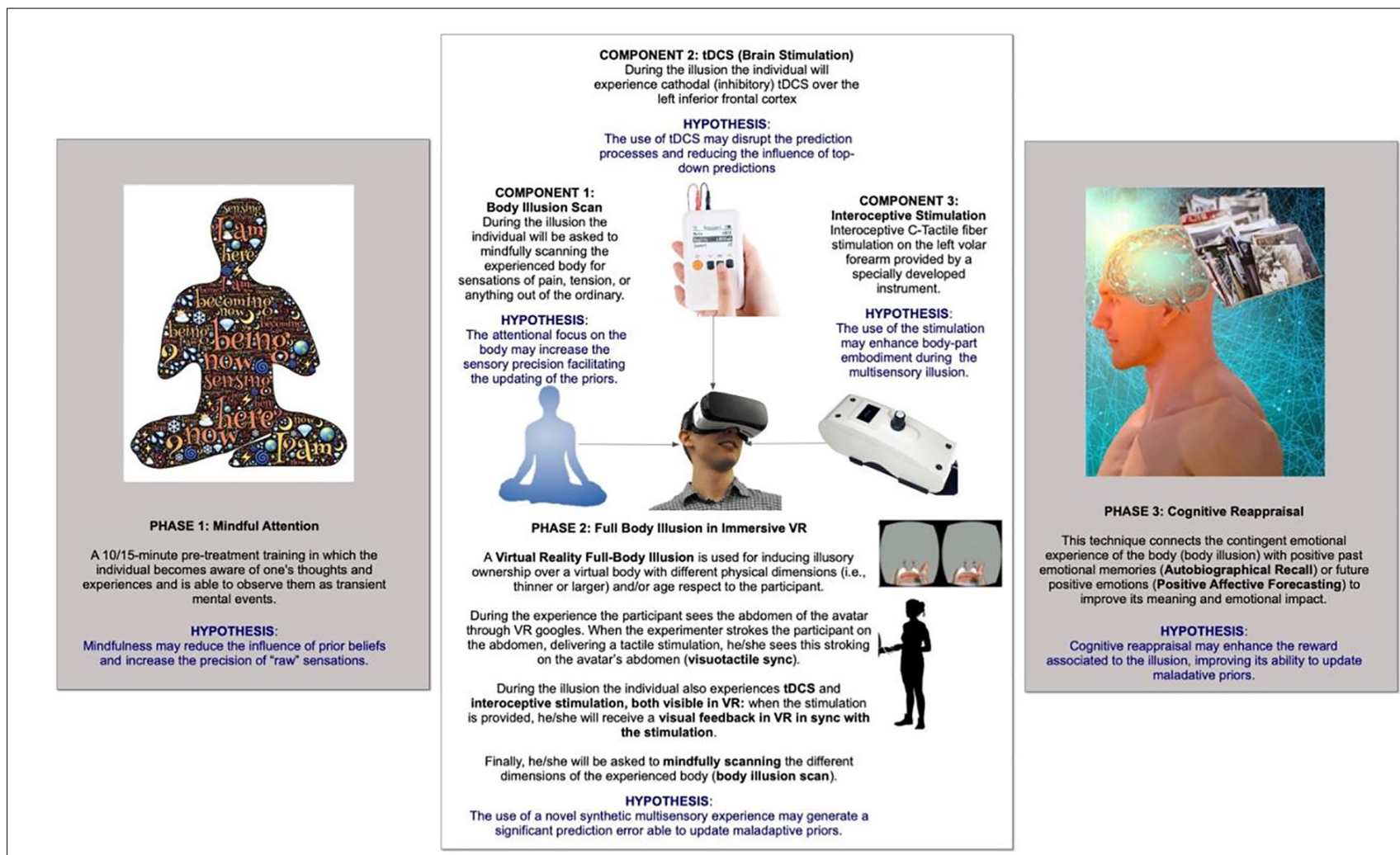

FIGURE 2 | Regenerative virtual therapy. 
Mindful attention is a primary component of mindfulness, which can be considered the awareness of being in the present moment without the burden of previous, mindless schemas (Pagnini and Philips, 2015). In Bayesian terms, this suggests that mindfulness may reduce the influence of prior beliefs and increase the precision of "raw" sensations (Manjaly and Iglesias, 2020). Preliminary findings supporting this hypothesis suggest that automatic reactions and behaviors (Papies et al., 2015), such as salivation following food conditioning (Baquedano et al., 2017), can be hindered with mindful attention.

4. The use of cognitive reappraisal to reconstruct and reelaborate the emotional content of the multisensory experience to improve its level of reward: specifically, we planned to increase the level of reward by connecting the body illusion with positive past emotional memories [i.e., autobiographical recall (AR)] or future positive emotions [i.e., positive affective forecasting (PAF)] to improve its meaning and emotional impact. AR connects the contingent emotional experience of the body with past emotional memories of it (Robinson, 1986; Mills and D'Mello, 2014). Instead, PAF connects the body illusion to how the individual will feel in the future.

\section{CONCLUSION}

This perspective introduced the RVT, a new therapeutical approach that wants to address a critical feature of most, if not all, psychopathologies: schema rigidity (Morris and Mansell, 2018). According to a predictive brain neuroscientific approach, mental disorders can be broadly characterized by a dysfunction in the way the brain computes and integrates the representations of the inner and outer body across time (i.e., BSC). Specifically, inaccurate or inflexible predictions can disturb the coherent integration of bodily and visceral signals and disrupt the optimal interaction of an individual with the external and social world.

In this view, the main goal of RVT was to allow a potential revision and de-weighting of maladaptive predictions through the integration of different technology-based somatic modification techniques with mindfulness and cognitive reappraisal.

The perspective discussed the rationale of this approach and presented a specific strategy based on the following steps:

- The development of a synthetic multisensory experience (i.e., visuotactile and interoceptive) to generate significant prediction errors: a virtual reality full-body illusion in sync with an interoceptive modulation characterized by a low level of predictability.

- The use of brain stimulation techniques to reduce the influence of top-down predictions.

- The use of mindfulness attention to improving the precision of the multisensory experience.

- The use of cognitive reappraisal to reconstruct and re-elaborate the emotional content of the multisensory experience to improve its level of reward.
On the one hand, this framework is based on a clear rationale and allows the identification of different hypotheses (presented in Figure 2) that can be tested experimentally. On the other hand, the clinical testing of the different assumptions is not easy, not only experimentally but also technically and computationally. The biggest challenge is the complexity of the different multisensory bodily experiences to be developed that involve both internal and external signals and both somatic and semantic/metacognitive domains.

Moreover, the closed-loop nature of BSC means that a modification in one domain typically invokes a cascade of changes throughout the different layers, making it difficult to differentiate cause from consequence. This suggests that, on the one hand, it is complex to evaluate the effects of the treatment given the many variables involved. On the other hand, the regulation of bodily variables through homeostasis and allostasis makes particularly challenging to determine whether problems of a patient originate in inference problems, regulation problems, or actual bodily dyshomeostasis as these can all lead to one another (Petzschner et al., 2017).

Finally, the number of somatic perturbation techniques that can be used to generate prediction errors is actually limited to the ones described in the study. As underlined by Petzschner et al. (2017), developing new tools that are non-invasive and provide temporal control is critical for the future of RVT. Moreover, the increasing availability of tools that allow the acquisition and (computational) analysis of neuroimaging and behavioral data may facilitate the validation of the model (Frässle et al., 2021).

In conclusion, RVT offers an empirically testable and potentially clinically useful framework that can improve the existing state-of-the-art in different ground-breaking ways, allowing us as follows:

- to acquire an unprecedented experimental overview of the dynamics of our bodily representations.

- to explain how somatic processes affect mental health and wellbeing.

- to reverse-engineer their functioning and hacking them using interoceptive and multisensory technologies.

\section{DATA AVAILABILITY STATEMENT}

The original contributions presented in the study are included in the article/supplementary material, further inquiries can be directed to the corresponding author/s.

\section{AUTHOR CONTRIBUTIONS}

GR conceived and developed the initial draft. FP revised the initial draft. SS, DD, and FP worked with GR to enhance the revised draft and develop it into the final draft. All authors have reviewed and approved the final manuscript as submitted. 


\section{REFERENCES}

Apps, M. A., and Tsakiris, M. (2014). The free-energy self: a predictive coding account of self-recognition. Neurosci. Biobehav. Rev. 41, 85-97. doi: 10.1016/ j.neubiorev.2013.01.029

Avenanti, A., Paracampo, R., Annella, L., Tidoni, E., and Aglioti, S. M. (2018). Boosting and decreasing action prediction abilities through excitatory and inhibitory tDCS of inferior frontal cortex. Cereb. Cortex 28, 1282-1296. doi: 10.1093/cercor/bhx041

Baquedano, C., Vergara, R., Lopez, V., Fabar, C., Cosmelli, D., and Lutz, A. (2017). Compared to self-immersion, mindful attention reduces salivation and automatic food bias. Sci. Rep. 7:13839. doi: 10.1038/s41598-017-13662-z

Barca, L., and Pezzulo, G. (2020). Keep your interoceptive streams under control: an active inference perspective on anorexia nervosa. Cogn. Affect. Behav. Neurosci. 20, 427-440. doi: 10.3758/s13415-020-00777-6

Barrett, L. F., Quigley, K. S., and Hamilton, P. (2016). An active inference theory of allostasis and interoception in depression. Philos. Trans. R. Soc. B Biol. Sci. 371:20160011. doi: 10.1098/rstb.2016.0011

Bennett, M. P., Knight, R., Patel, S., So, T., Dunning, D., Barnhofer, T., et al. (2021). Decentering as a core component in the psychological treatment and prevention of youth anxiety and depression: a narrative review and insight report. Transl. Psychiatry 11:288. doi: 10.1038/s41398-021-01397-5

Björnsdotter, M., Morrison, I., and Olausson, H. (2010). Feeling good: on the role of C fiber mediated touch in interoception. Exp. Brain Res. 207, 149-155. doi: 10.1007/s00221-010-2408-y

Blanke, O. (2012). Multisensory brain mechanisms of bodily self-consciousness. Nat. Rev. Neurosci. 13, 556-571. doi: 10.1038/nrn3292

Blanke, O., Slater, M., and Serino, A. (2015). Behavioral, neural, and computational principles of bodily self-consciousness. Neuron 88, 145-166. doi: 10.1016/j. neuron.2015.09.029

Botvinick, M., and Cohen, J. (1998). Rubber hands 'feel' touch that eyes see. Nature 391:756. doi: 10.1038/35784

Browning, M., Carter, C. S., Chatham, C., Den Ouden, H., Gillan, C. M., Baker, J. T., et al. (2020). Realizing the clinical potential of computational psychiatry: report from the banbury center meeting, february 2019. Biol. Psychiatry 88, e5-e10. doi: 10.1016/j.biopsych.2019.12.026

Clark, A. (2013). Whatever next? Predictive brains, situated agents, and the future of cognitive science. Behav. Brain Sci. 36, 181-204. doi: 10.1017/ S0140525X12000477

Clark, A. (2016b). Surfing Uncertainty: Prediction, Action, And The Embodied Mind. Oxford: Oxford University Press. doi: 10.1093/acprof: oso/9780190217013.001.0001

Clark, A. (2016a). Attention alters predictive processing. Behav. Brain Sci. 39:e234. doi: 10.1017/S0140525X15002472

Dakanalis, A., Gaudio, S., Serino, S., Clerici, M., Carrà, G., and Riva, G. (2016). Body-image distortion in anorexia nervosa. Nat. Rev. Dis. Primers 2:16026. doi: 10.1038/nrdp.2016.26

Di Lernia, D., Cipresso, P., Pedroli, E., and Riva, G. (2018). Toward an embodied medicine: a portable device with programmable interoceptive stimulation for heart rate variability enhancement. Sensors (Basel) 18:2469. doi: 10.3390/ s18082469

Di Lernia, D., Lacerenza, M., Ainley, V., and Riva, G. (2020). Altered interoceptive perception and the effects of interoceptive analgesia in musculoskeletal, primary, and neuropathic chronic pain conditions. J. Pers. Med. 10:201. doi: 10.3390/jpm10040201

Di Lernia, D., Serino, S., and Riva, G. (2016). Pain in the body. Altered interoception in chronic pain conditions: a systematic review. Neurosci. Biobehav. Rev. 71, 328-341. doi: 10.1016/j.neubiorev.2016.09.015

Ferri, F., Nikolova, Y. S., Perrucci, M. G., Costantini, M., Ferretti, A., Gatta, V., et al. (2017). A neural "tuning curve" for multisensory experience and cognitive-perceptual schizotypy. Schizophr. Bull. 43, 801-813. doi: 10.1093/ schbul/sbw174

Frässle, S., Aponte, E. A., Bollmann, S., Brodersen, K. H., Do, C. T., Harrison, O. K., et al. (2021). TAPAS: an open-source software package for translational neuromodeling and computational psychiatry. Front. Psychiatry 12:680811. doi: 10.3389/fpsyt.2021.680811

Freeman, D., Reeve, S., Robinson, A., Ehlers, A., Clark, D., Spanlang, B., et al. (2017). Virtual reality in the assessment, understanding, and treatment of mental health disorders. Psychol. Med. 47, 2393-2400. doi: 10.1017/ S003329171700040X

Friston, K. J. (2010). The free-energy principle: a unified brain theory? Nat. Rev. Neurosci. 11, 127-138. doi: 10.1038/nrn2787

Friston, K. J., Stephan, K. E., Montague, R., and Dolan, R. J. (2014). Computational psychiatry: the brain as a phantastic organ. Lancet Psychiatry 1, 148-158. doi: 10.1016/S2215-0366(14)70275-5

Gaggioli, A., and Riva, G. (2014). Psychological treatments: smart tools boost mental-health care. Nature 512:28. doi: 10.1038/512028b

Gallace, A., and Spence, C. (2014). In Touch With The Future: The Sense of Touch From Cognitive Neuroscience to Virtual Reality. Oxford: Oxford University Press. doi: 10.1093/acprof:oso/9780199644469.001.0001

Henriques, D. Y., and Cressman, E. K. (2012). Visuomotor adaptation and proprioceptive recalibration. J. Mot. Behav. 44, 435-444. doi: 10.1080/ 00222895.2012.659232

Holmes, E. A., Craske, M. G., and Graybiel, A. M. (2014). Psychological treatments: a call for mental-health science. Nature 511, 287-289. doi: 10.1038/511287a

Iodice, P., Porciello, G., Bufalari, I., Barca, L., and Pezzulo, G. (2019). An interoceptive illusion of effort induced by false heart-rate feedback. Proc. Natl. Acad. Sci. U.S.A. 116, 13897-13902. doi: 10.1073/pnas.1821032116

Keizer, A., Smeets, M. A., Dijkerman, H. C., Uzunbajakau, S. A., van Elburg, A., and Postma, A. (2013). Too fat to fit through the door: first evidence for disturbed body-scaled action in anorexia nervosa during locomotion. PLoS One 8:e64602. doi: 10.1371/journal.pone.0064602

Keizer, A., van Elburg, A., Helms, R., and Dijkerman, H. C. (2016). A virtual reality full body illusion improves body image disturbance in anorexia nervosa. PLoS One 11:e0163921. doi: 10.1371/journal.pone.0163921

Klaver, M., and Dijkerman, H. C. (2016). Bodily experience in schizophrenia: factors underlying a disturbed sense of body ownership. Front. Hum. Neurosci. 10:305. doi: 10.3389/fnhum.2016.00305

Lux, V., Non, A. L., Pexman, P. M., Stadler, W., Weber, L. A. E., and Krüger, M. (2021). A developmental framework for embodiment research: the next step toward integrating concepts and methods. Front. Syst. Neurosci. 15:672740. doi: $10.3389 /$ fnsys. 2021.672740

Mahla, R. S. (2016). Stem cells applications in regenerative medicine and disease therapeutics. Int. J. Cell Biol. 2016:6940283. doi: 10.1155/2016/6940283

Maister, L., Slater, M., Sanchez-Vives, M. V., and Tsakiris, M. (2015). Changing bodies changes minds: owning another body affects social cognition. Trends Cogn. Sci. 19, 6-12. doi: 10.1016/j.tics.2014.11.001

Mancuso, V., Stramba-Badiale, C., Cavedoni, S., Pedroli, E., Cipresso, P., and Riva, G. (2020). Virtual reality meets non-invasive brain stimulation: integrating two methods for cognitive rehabilitation of mild cognitive impairment. Front. Neurol. 11:566731. doi: 10.3389/fneur.2020.566731

Manjaly, Z.-M., and Iglesias, S. (2020). A Computational theory of mindfulness based cognitive therapy from the "bayesian brain" perspective. Front. Psychiatry 11:404. doi: 10.3389/fpsyt.2020.00404

Marotta, A., Re, A., Zampini, M., and Fiorio, M. (2021). Bodily self-perception during voluntary actions: the causal contribution of premotor cortex and cerebellum. Cortex 142, 1-14. doi: 10.1016/j.cortex.2021.05.012

Mason, C., and Dunnill, P. (2008). A brief definition of regenerative medicine. Regen. Med. 3, 1-5. doi: 10.2217/17460751.3.1.1

Matamala-Gomez, M., Maselli, A., Malighetti, C., Realdon, O., Mantovani, F., and Riva, G. (2021). Virtual body ownership illusions for mental health: a narrative review. J. Clin. Med. 10:139. doi: 10.3390/jcm10010139

McGuire, J. T., Nassar, M. R., Gold, J. I., and Kable, J. W. (2014). Functionally dissociable influences on learning rate in a dynamic environment. Neuron 84, 870-881. doi: 10.1016/j.neuron.2014.10.013

Mills, C., and D'Mello, S. (2014). On the validity of the autobiographical emotional memory task for emotion induction. PLoS One 9:e95837. doi: 10.1371/journal. pone.0095837

Mirza, M. B., Adams, R. A., Friston, K., and Parr, T. (2019). Introducing a bayesian model of selective attention based on active inference. Sci. Rep. 9:13915. doi: 10.1038/s41598-019-50138-8

Möller, T. J., Georgie, Y. K., Schillaci, G., Voss, M., Hafner, V. V., and Kaltwasser, L. (2021). Computational models of the "active self" and its disturbances in schizophrenia. Conscious. Cogn. 93:103155. doi: 10.1016/j.concog.2021.103155

Morris, L., and Mansell, W. (2018). A systematic review of the relationship between rigidity/flexibility and transdiagnostic cognitive and behavioral processes that 
maintain psychopathology. J. Exp. Psychopathol. 9:2043808718779431. doi: $10.1177 / 2043808718779431$

Moseley, G. L., Gallace, A., and Spence, C. (2012). Bodily illusions in health and disease: physiological and clinical perspectives and the concept of a cortical 'body matrix'. Neurosci. Biobehav. Rev. 36, 34-46. doi: 10.1016/j.neubiorev. 2011.03.013

Nair, A., Rutledge, R. B., and Mason, L. (2020). Under the hood: using computational psychiatry to make psychological therapies more mechanismfocused. Front. Psychiatry 11:140. doi: 10.3389/fpsyt.2020.00140

Neuser, M. P., Teckentrup, V., Kuhnel, A., Hallschmid, M., Walter, M., and Kroemer, N. B. (2020). Vagus nerve stimulation boosts the drive to work for rewards. Nat. Commun. 11:3555. doi: 10.1038/s41467-020-17344-9

Newen, A. (2018). The embodied self, the pattern theory of self, and the predictive mind. Front. Psychol. 9:2270. doi: 10.3389/fpsyg.2018.02270

Nourouzpour, N., Salomonczyk, D., Cressman, E. K., and Henriques, D. Y. (2015). Retention of proprioceptive recalibration following visuomotor adaptation. Exp. Brain Res. 233, 1019-1029. doi: 10.1007/s00221-014-4176-6

Owens, A. P., Allen, M., Ondobaka, S., and Friston, K. J. (2018). Interoceptive inference: from computational neuroscience to clinic. Neurosci. Biobehav. Rev. 90, 174-183. doi: 10.1016/j.neubiorev.2018.04.017

Pagnini, F., and Philips, D. (2015). Being mindful about mindfulness. Lancet Psychiatry 2, 288-289. doi: 10.1016/S2215-0366(15)00041-3

Papies, E. K., Pronk, T. M., Keesman, M., and Barsalou, L. W. (2015). The benefits of simply observing: mindful attention modulates the link between motivation and behavior. J. Pers. Soc. Psychol. 108, 148-170. doi: 10.1037/a0038032

Park, H.-D., and Blanke, O. (2019). Coupling inner and outer body for selfconsciousness. Trends Cogn. Sci. 23, 377-388. doi: 10.1016/j.tics.2019.02.002

Paulus, M. P., Feinstein, J. S., and Khalsa, S. S. (2019). An active inference approach to interoceptive psychopathology. Annu. Rev. Clin. Psychol. 15, 97-122. doi: 10.1146/annurev-clinpsy-050718-095617

Petzschner, F. H., Weber, L. A. E., Gard, T., and Stephan, K. E. (2017). Computational psychosomatics and computational psychiatry: toward a joint framework for differential diagnosis. Biol. Psychiatry 82, 421-430. doi: 10.1016/ j.biopsych.2017.05.012

Pezzulo, G., Rigoli, F., and Friston, K. (2015). Active Inference, homeostatic regulation and adaptive behavioural control. Prog. Neurobiol. 134, 17-35. doi: 10.1016/j.pneurobio.2015.09.001

Pollatos, O., Herbert, B. M., Mai, S., and Kammer, T. (2016). Changes in interoceptive processes following brain stimulation. Philos. Trans. R. Soc. B Biol. Sci. 371:20160016. doi: 10.1098/rstb.2016.0016

Ponzo, S., Kirsch, L. P., Fotopoulou, A., and Jenkinson, P. M. (2018). Balancing body ownership: visual capture of proprioception and affectivity during vestibular stimulation. Neuropsychologia 117, 311-321. doi: 10.1016/j. neuropsychologia.2018.06.020

Ponzo, S., Kirsch, L. P., Fotopoulou, A., and Jenkinson, P. M. (2019). Vestibular modulation of multisensory integration during actual and vicarious tactile stimulation. Psychophysiology 56:e13430. doi: 10.1111/psyp.13430

Postmes, L., Sno, H. N., Goedhart, S., van der Stel, J., Heering, H. D., and de Haand, L. (2014). Schizophrenia as a self-disorder due to perceptual incoherence. Schizophr. Res. 152, 41-50. doi: 10.1016/j.schres.2013.07.027

Riva, G. (2018). The neuroscience of body memory: from the self through the space to the others. Cortex 104, 241-260. doi: 10.1016/j.cortex.2017.07.013

Riva, G., and Dakanalis, A. (2018). Altered processing and integration of multisensory bodily representations and signals in eating disorders: a possible path toward the understanding of their underlying causes. Front. Hum. Neurosci. 12:49. doi: 10.3389/fnhum.2018.00049

Riva, G., and Gaudio, S. (2012). Allocentric lock in anorexia nervosa: new evidences from neuroimaging studies. Med. Hypotheses 79, 113-117. doi: 10.1016/j.mehy. 2012.03.036

Riva, G., Di Lernia, D., and Dakanalis, A. (2019a). Being socially uninterested versus not having social prediction skills: the impact of multisensory integration deficits on social skills in autism. Behav. Brain Sci. 42:e109. doi: 10.1017/ S0140525X18002340
Riva, G., Wiederhold, B. K., and Mantovani, F. (2019b). Neuroscience of virtual reality: from virtual exposure to embodied medicine. Cyberpsychol. Behav. Soc. Netw. 22, 82-96. doi: 10.1089/cyber.2017.29099.gri

Robinson, J. A. (1986). “Autobiographical memory: a historical prologue,"in Autobiographical Memory, Vol. 325, ed. D. C. Rubin (Cambridge: Cambridge University Press), 19-24. doi: 10.1017/CBO9780511558313.005

Ropar, D., Greenfield, K., Smith, A. D., Carey, M., and Newport, R. (2018). Body representation difficulties in children and adolescents with autism may be due to delayed development of visuo-tactile temporal binding. Dev. Cogn. Neurosci. 29, 78-85. doi: 10.1016/j.dcn.2017.04.007

Scarpina, F., Migliorati, D., Marzullo, P., Mauro, A., Scacchi, M., and Costantini, M. (2016). Altered multisensory temporal integration in obesity. Sci. Rep. 6:28382. doi: $10.1038 /$ srep 28382

Schoeller, F., Haar, A. J. H., Jain, A., and Maes, P. (2019). Enhancing human emotions with interoceptive technologies. Phys. Life Rev. 31, 310-319. doi: 10.1016/j.plrev.2019.10.008

Sedda, A., Tonin, D., Salvato, G., Gandola, M., and Bottini, G. (2016). Left caloric vestibular stimulation as a tool to reveal implicit and explicit parameters of body representation. Conscious. Cogn. 41, 1-9. doi: 10.1016/j.concog.2016.01.012

Smout, C. A., Tang, M. F., Garrido, M. I., and Mattingley, J. B. (2019). Attention promotes the neural encoding of prediction errors. PLoS Biol. 17:e2006812. doi: 10.1371/journal.pbio.2006812

Stephan, K. E., Manjaly, Z. M., Mathys, C. D., Weber, L. A. E., Paliwal, S., Gard, T., et al. (2016). Allostatic self-efficacy: a metacognitive theory of dyshomeostasisinduced fatigue and depression. Front.Hum. Neurosci. 10:550. doi: 10.3389/ fnhum.2016.00550

Stramba-Badiale, C., Mancuso, V., Cavedoni, S., Pedroli, E., Cipresso, P., and Riva, G. (2020). Transcranial magnetic stimulation meets virtual reality: the potential of integrating brain stimulation with a simulative technology for food addiction. Front. Neurosci. 14:720. doi: 10.3389/fnins.2020.00720

Tajadura-Jiménez, A., and Tsakiris, M. (2014). Balancing the "inner" and the "outer" self: interoceptive sensitivity modulates self-other boundaries. J. Exp. Psychol. Gen. 143, 736-744. doi: 10.1037/a0033171

Talsma, D. (2015). Predictive coding and multisensory integration: an attentional account of the multisensory mind. Front. Integr. 9:19. doi: 10.3389/fnint.2015. 00019

Tatti, E., Rossi, S., Innocenti, I., Rossi, A., and Santarnecchi, E. (2016). Non-invasive brain stimulation of the aging brain: state of the art and future perspectives. Ageing Res. Rev. 29, 66-89. doi: 10.1016/j.arr.2016.05.006

Tsay, A., Allen, T. J., Proske, U., and Giummarra, M. J. (2015). Sensing the body in chronic pain: a review of psychophysical studies implicating altered body representation. Neurosci. Biobehav. Rev. 52, 221-232. doi: 10.1016/j.neubiorev. 2015.03.004

Wiederhold, B. K., and Riva, G. (2019). Virtual reality therapy: emerging topics and future challenges. Cyberpsychol. Behav. Soc. Netw. 22, 3-6. doi: 10.1089/cyber. 2018.29136.bkw

Conflict of Interest: The authors declare that the research was conducted in the absence of any commercial or financial relationships that could be construed as a potential conflict of interest.

Publisher's Note: All claims expressed in this article are solely those of the authors and do not necessarily represent those of their affiliated organizations, or those of the publisher, the editors and the reviewers. Any product that may be evaluated in this article, or claim that may be made by its manufacturer, is not guaranteed or endorsed by the publisher.

Copyright (C) 2021 Riva, Serino, Di Lernia and Pagnini. This is an open-access article distributed under the terms of the Creative Commons Attribution License (CC BY). The use, distribution or reproduction in other forums is permitted, provided the original author(s) and the copyright owner(s) are credited and that the original publication in this journal is cited, in accordance with accepted academic practice. No use, distribution or reproduction is permitted which does not comply with these terms. 